\title{
High-Resolution Multifocal Pupillographic Objective Perimetry in Glaucoma
}

\author{
Corinne F. Carle, ${ }^{1}$ Andrew C. James, ${ }^{1}$ Maria Kolic, ${ }^{1,2}$ Yik-Wen Lob, ${ }^{1}$ and Ted Maddess ${ }^{1}$
}

\begin{abstract}
Purpose. The recent development of an objective and noninvasive perimetric technique using pupillary responses to sparse multifocal visual stimuli shows promise for the assessment of visual function in glaucoma. This study assesses the sensitivity and specificity of four variants of dichoptic multifocal pupillographic objective perimetry (mfPOP) with a highresolution, 40-region/field stimulus.
\end{abstract}

Methods. Nineteen normal subjects and 17 with open-angle glaucoma were tested with four 4-minute stimulus protocols, presented in eight segments of 30 seconds each. Achromatic multifocal stimuli comprised 40 test regions per eye arranged in a four-ring dartboard layout subtending $60^{\circ}$ of visual field. Background luminance was $10 \mathrm{~cd} / \mathrm{m}^{2}$ with active stimulus regions displaying steady or flickered stimuli at $290 \mathrm{~cd} / \mathrm{m}^{2}$. Stimulus durations were between 33 and 150 ms, mean intervals between presentations to each test region ranged from 1 to 16 seconds. Fixation was monitored in real time.

RESULTS. Longer mean intervals and durations resulted in better diagnostic performance. Best results were obtained with 150-ms flickered stimuli and a discriminant function that incorporated both amplitude and width of responses: ROC area under the curve $0.86 \pm 0.05$ (mean $\pm \mathrm{SE}$ ) across all visual field severities, $(n=34)$ and $1.00 \pm 0.00$ for moderate and severe fields $(n=10)$.

Conclusions. mfPOP produces separate information on response delay and afferent and efferent defects at every point in the field. The diagnostic accuracy of the 40-region, 150-ms stimulus is comparable to that of commonly used subjective perimeters and encourages further investigation of this technique. (Invest Opbthalmol Vis Sci. 2011;52:604-610) DOI:10.1167/iovs.10-5737

$\mathrm{T}$ he first indication of glaucoma may be evidence of abnormalities in either optic disc morphology or visual function. ${ }^{1}$ The availability of tools with the ability to provide early, independent, and reliable assessment of these signs is therefore crucial to the effective diagnosis of this optic neuropathy. After a diagnosis, the subsequent management of glaucoma is heavily reliant on the accurate monitoring of progression, since determining the efficacy of a particular treatment is problematic if

From the ${ }^{1}$ ARC (Australian Research Council) Centre of Excellence in Vision Science, Research School of Biology, The Australian National University, Canberra, Australia.

${ }^{2}$ Present affilation: Seeing Machines Limited, Canberra, Australia. Supported by Grant CE0561903 from the ARC through the ARC Centre of Excellence in Vision Science.

Submitted for publication April 19, 2010; revised July 20, 2010; accepted September 9, 2010.

Disclosure: C.F. Carle, Seeing Machines (F); A.C. James, Seeing Machines (F, I, C, R), P; M. Kolic, Seeing Machines (F, E, R); Y.-W. Loh, Seeing Machines (F); T. Maddess, Seeing Machines (F, I, C, R), P Corresponding author: Corinne F. Carle, Research School of Biology, The Australian National University, GPO Box 475, Canberra, ACT 2601, Australia; corinne.carle@anu.edu.au. its effects cannot be reliably measured. At present, many forms of perimetry require lengthy test durations. High test-retest variability is also a problem, often being most pronounced in regions of the visual field with impaired function. ${ }^{2-7}$ This variablity causes difficulties in the accurate assessment of disease progression and requires the use of more and longer tests. The reliance of many of these perimeters on subjective patient responses possibly contributes to these problems; therefore, objective tests may have advantages. Unfortunately, currently available forms of objective perimetry such as multifocal visual evoked potentials (mfVEPs) and multifocal electroretinography (mfERG), can be somewhat invasive, lengthy, and complicated to administer. ${ }^{2}$ It is apparent that the development of a fast and objective, noncontact perimetric test is a highly desirable goal.

Several studies have explored perimetry based on pupillary responses as a means of developing such a test. ${ }^{8-12}$ These methods have been hampered, however, by long test durations. Recently, multifocal techniques developed for mfERGs ${ }^{13}$ have been applied to pupillography. ${ }^{14,15}$ In our research, this method has involved the use of multifocal stimuli that are both temporally and spatially sparse, an innovation that has been shown to enhance signal-to-noise ratios (SNRs) in mfVEPs. ${ }^{16-21}$ The promising results obtained in these early studies, with stimuli that tested 24 regions within each $60^{\circ}$ visual field, ${ }^{22}$ suggest that it may be possible to employ a larger number of smaller sized test regions. This method would provide higher resolution of the visual fields while maintaining a test duration of only 4 minutes for both eyes. To this end, we sought to assess the diagnostic accuracy of four variants of mfPOP using a high-resolution stimulus layout with 40 test regions per eye.

\section{Methods}

Thirty-six subjects were tested with four variants of mfPOP, having had their diagnostic status determined within 6 months of this study with Humphrey (HFA II) Achromatic SITA-Fast perimetry, SWAP Fastpac 24-2 perimetry, Matrix 24-2 perimetry, and Stratus OCT (all from Carl Zeiss Meditec Inc., Dublin, CA); slit-lamp biomicroscopy; and applanation tonometry. The study groups consisted of the following: the glaucoma group, 10 women, 7 men, aged $61.5 \pm 9.7$ years, and the normal group, 11 women, 8 men, aged $59.7 \pm 8.7$ years. Both eyes of each subject were tested concurrently ( $n=72$ fields). Informed written consent was given by all participants after explanation of the nature and possible consequences of the study, according to the ANU Human Experimentation Ethics Committee approval 238/04. All research adhered to the tenets of the Declaration of Helsinki.

Glaucoma subjects required a diagnosis of open-angle glaucoma with evidence of glaucomatous scotomas in at least one eye (three had diagnoses of normal-tension glaucoma). Functional defects in each eye of glaucoma subjects were classified based on the HFA mean deviation (MD) as follows: severe, $\mathrm{MD}<-12 \mathrm{~dB}(n=4$ eyes $)$; moderate, $\mathrm{MD}<$ $-6 \mathrm{~dB}$ but $\geq-12 \mathrm{~dB}$ ( $n=6$ eyes); mild, MD $\geq-6 \mathrm{~dB}$ ( $n=22$ eyes); and ND, no apparent field defects ( $n=2$ fellow eyes). Normal subjects were required to have no detectable glaucomatous abnormalities, open angles, discs within normal limits, and intraocular pressure $<21 \mathrm{~mm}$ 


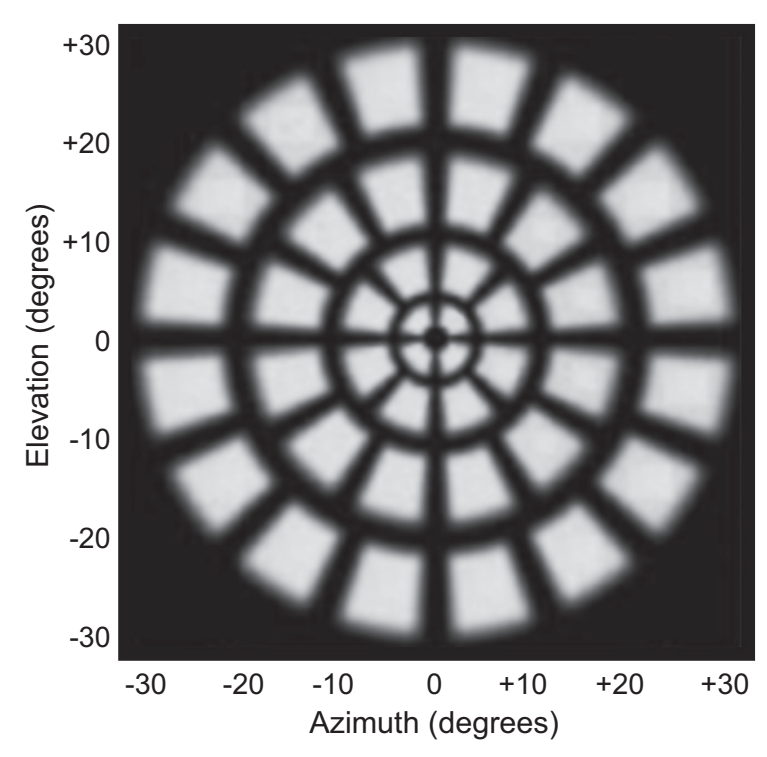

FIGURE 1. T30-40 stimulus map showing scaling of regions according to eccentricity. The stimuli are shown as if all regions were on at the same time, which would not happen in practice. The stimuli edges were blurred to reduce the effects of misrefraction.

Hg. Exclusion criteria for both groups included the presence of other ocular disease or previous ocular surgery (with the exception of argon or selective laser trabeculoplasty), refractive errors greater than $\pm 6 \mathrm{D}$ or more than $2 \mathrm{D}$ of cylinder, or current use of miotic medication. Subjects were requested not to consume caffeine or alcohol for 1 hour before testing.

\section{Stimuli}

The T30-40 stimulus layout used in this experiment subtended $60^{\circ}$ of visual angle $\left( \pm 30^{\circ}\right)$ and consisted of 40 test regions arranged in a dartboard layout (Fig. 1). Responses to possible light scatter from adjacent regions were reduced by the noncontiguous layout of the regions and the $10-\mathrm{cd} / \mathrm{m}^{2}$ background illumination. Subjects in both groups underwent a randomly ordered series of four stimulus protocols, each consisting of eight segments of 30 seconds' duration (Table 1). In the first of these, the test regions of the less sparse steady (LSS) protocol, when active, displayed a single $33-\mathrm{ms}$, steady $290 \mathrm{~cd} / \mathrm{m}^{2}$ stimulus pulse, with activations in each region separated by a mean interval of 1 second. Active regions in the other three protocols, less sparse flicker (LSF), moderately sparse flicker (MSF), and very sparse flicker (VSF), displayed $20-\mathrm{Hz}$ flickered modulation between 10 and $290 \mathrm{~cd} / \mathrm{m}^{2}$.

TABle 1. Temporal and Luminance Characteristics of the Four Stimulus Protocols

\begin{tabular}{|c|c|c|c|c|}
\hline & LSS & LSF & MSF & VSF \\
\hline Flicker, Hz & 0 & 20 & 20 & 20 \\
\hline Stimulus duration, ms & 33 & 100 & 100 & 150 \\
\hline $\begin{array}{l}\text { Mean interval between stimulus } \\
\text { presentations to each test region, } s\end{array}$ & 1 & 1 & 4 & 16 \\
\hline
\end{tabular}

All stimuli were white and were presented at $290 \mathrm{~cd} / \mathrm{m}^{2}$ on a $10-\mathrm{cd} / \mathrm{m}^{2}$ background. The LSS stimulus protocol consisted of steady stimuli, all other protocols utilized stimuli that flickered at $20 \mathrm{~Hz}$. Stimulus durations varied from $33 \mathrm{~ms}$ in the LSS and LSF protocols to $150 \mathrm{~ms}$ in the VSF. Temporal sparseness of stimulus presentations in each region also differed, with mean intervals ranging between 1 and 16 seconds. The number of presentations made to each region can be obtained by dividing the total test time of 240 seconds by the mean interval between stimulus presentations.
Flicker was generated by displaying a bright stimulus for one frame, then two dark frames; the period for decay of the LCD display to $1 / e$ was approximately $10 \mathrm{~ms}$. The duration of these flickered stimuli was either 100 or $150 \mathrm{~ms}$, and the mean presentation intervals within each individual region were 1,4 , or 16 seconds.

\section{Multifocal Infrared Pupillography}

Presentation of stimuli and monitoring of pupil diameter was performed using a prototype of the FD-cleared Truefield Analyzer (Seeing Machines Ltd., Braddon, ACT, Australia). This device, under development at the time of this experiment, utilizes concurrent presentation of dichoptic, multifocal stimuli at 60 frames/second (Fig. 2). Infrared light was used to illuminate the subjects' pupils, and their responses were monitored with two video cameras at 30 frames/second/eye, synchronous with stimulus presentation. During testing, subjects fixated a small red cross in the center of the viewing field. Gaze was monitored online, and data from blinks and fixation losses were deleted. The system can tolerate up to $15 \%$ data loss before a given 30 -second segment must be repeated. Stimuli were presented at optical infinity to minimize accommodative responses. Corrective lenses compensated for refractive errors to within $1.5 \mathrm{D}$ of the subject's current optical prescription; the stimuli contained no spatial frequencies above 2 cyc/deg, making them highly tolerant of misrefraction. ${ }^{23}$

\section{Response Estimation}

All data analysis was performed using MatLab R2009b (The MathWorks, Natick, MA). Response waveforms for each test region were extracted from raw pupillary responses using a previously described

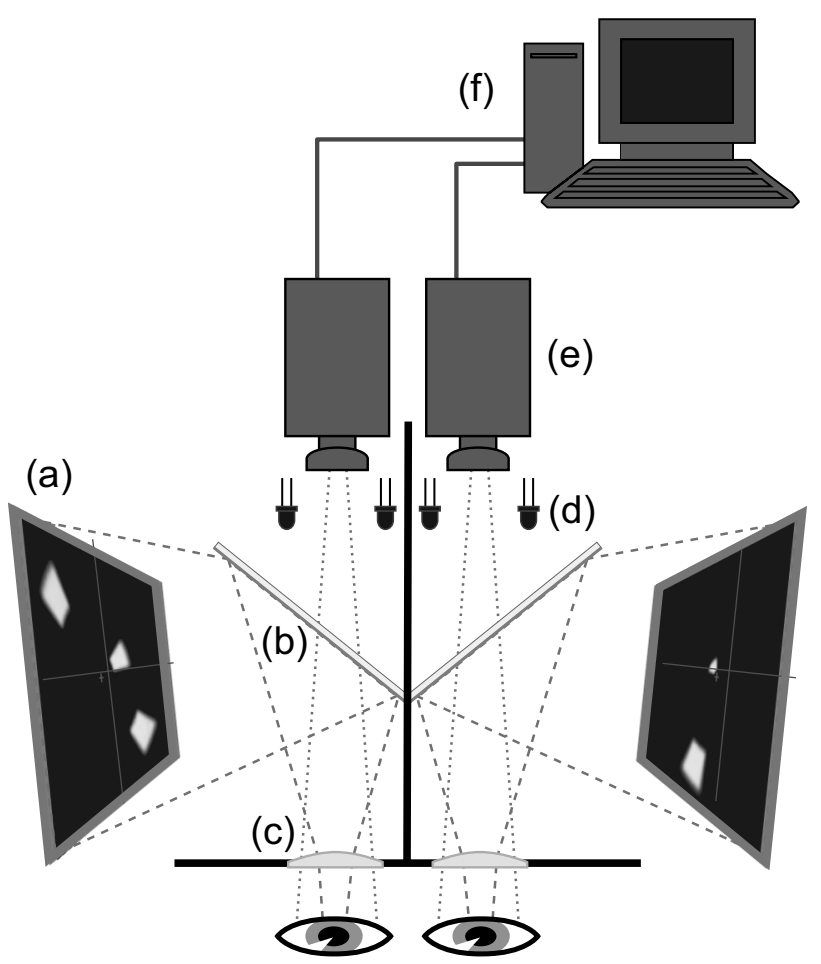

Figure 2. Measurement of pupillary responses using the Truefield Analyzer. Stimuli are presented independently on two LCD monitors (a). These images are reflected by two cold dichroic mirrors (b) allowing infrared light to pass while reflecting shorter wavelengths. Viewing distance is increased to optical infinity by plano convex lenses (c). Each eye views only one monitor, with the images being fused by the subject into a cyclopean view. Illumination of the eyes is provided by four infrared light-emitting diodes (d) facilitating the monitoring of each pupil by a separate infrared video camera (e). Pupil diameters are then extracted in real-time and retained by using a personal computer (f). (Not drawn to scale) 
multiple linear regression method developed originally for the analysis of mfVEPs. ${ }^{16,17}$ This process provided a set of 160 response estimates for each subject: both direct and consensual responses for left and right eyes for each of the 40 test regions. These estimated responses are effectively a mean impulse response obtained from 15 (VSF), 60 (MSF), or 240 (LSF and LSS) individual stimulus presentations to each region. While higher-order temporal and spatial interactions can be extracted with this method, only first-order components were found to be significant and so higher-order terms were not included. These measured responses were fitted with the lognormal function:

$$
v(t)=\exp \left\{\frac{-\left[\ln \left(t / t_{\mathrm{p}}\right)\right]^{2}}{2 \sigma^{2}}\right\}
$$

where $v(t)$ is the response waveform, $t$ defines the time at which each estimation is made, $t_{\mathrm{p}}$ is the time to peak, and $\sigma$ is the shape parameter. This calculation allowed the characterization of relevant response components according to the three measures of amplitude, width, and time to peak (Fig. 3). Width is the time in seconds between the points on the response waveform where $v(t)=1 / e$ (maximum amplitude).

Because of variations in mean pupil diameter in the general population, intersubject comparisons were facilitated by the standardization of contraction amplitudes, as follows:

$$
\text { AmpStd }=\text { contraction amplitude }(\mu \mathrm{m}) \times \frac{3500}{c}
$$

where $c$ is the mean value of a trend line through each subject's raw pupil diameter record. Therefore, this method normalized the pupil diameter and then multiplied that by the estimated population mean pupil diameter $(3500 \mu \mathrm{m})$ to provide units of micrometers for a typical subject. This method has been found to minimize many of the adverse effects of smaller or misshapen pupils. ${ }^{22,24} \mathrm{~A}$ check was performed by regression analysis to determine whether $c$ was subject to variation with either age or diagnostic status, two potential confounds for this method of standardization. This analysis revealed no significant relationships $(P>0.05)$ between mean pupil diameters and these two factors in this experiment.

\section{Receiver Operating Characteristic Analysis}

Receiver operating characteristic (ROC) analysis was used to establish which parameters were most effective at ascertaining the diagnostic status of subjects and to assess the diagnostic performance of each of the four protocols. The basic idea was to form regional deviations from normal responses that are similar to the total deviations measured on a perimeter and enter those deviations into the ROC analysis. The steps in the calculation of these deviations have been given in detail elsewhere. ${ }^{22,24}$ Conceptually, the steps were as follows: select a response variable (e.g., time to peak), pool the direct and consensual field data compute deviations from the region-wise normative data for all subjects, enter the deviations into the ROC analysis. The method of estimating deviations for normal subjects excluded each particular subject's results from the pooled normative data for that comparison, the so called leave-one-out method. ${ }^{25}$ Therefore, no subject was compared against the population that included their own results. No
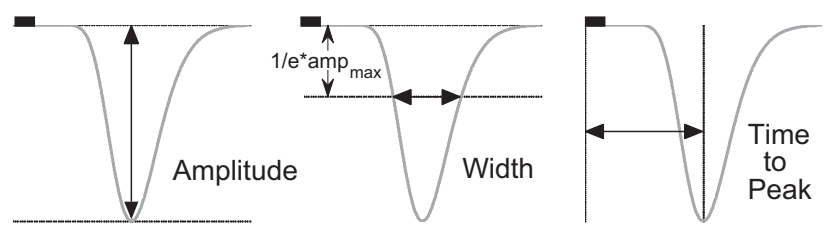

FIGURE 3. Extracted pupil responses for each test region were fitted with a lognormal function allowing characterization by amplitude, width, and time to peak. Short borizontal bars: the duration of a single stimulus pulse; double-headed arrow: parameter of interest. specific clustering of regions was performed; however, the total number of regions included in the analysis was varied, to examine the effect of the region number on accuracy. This provided a nonparametric means of comparing the diagnostic performance of the different protocols. More complex methods would require larger datasets for validation and therefore were not undertaken in this study.

\section{Results}

On average, $97.7 \% \pm 2.4 \%$ (SD) of data collected for each subject was able to be analyzed, with the remainder lost due to factors such as blinks or fixation losses. That being said the regression-based estimation of the responses from the pupil records meant that all data sets could be used. Mean test durations were similar across all protocols, ranging between 5 minutes $23 \pm 16$ seconds (SD) for the LSS protocol and 5 minutes $42 \pm 60$ seconds (SD) for the MSF protocol. Ninetyone percent of tests were completed within 6 minutes. Multivariate linear analysis, statistically adjusted to account for any correlation between paired eyes, demonstrated that pupillary response characteristics differed according to the stimulus protocol, diagnostic status and location of the stimulus region in the visual field. The largest contraction amplitudes were obtained in the temporal field in all stimulus protocols (Fig. 4). Protocols with more sparse presentation produced larger contraction amplitudes, the VSF protocol producing by far the largest. This protocol also elicited the highest mean values for width (Table 2B) and time to peak (Table 2C).

Mean response amplitudes of glaucoma patients were found to be between 1.2 and $12.2 \mu \mathrm{m}$ smaller than those of normal subjects (Table $2 \mathrm{~A}$ ). These effects were highly significant with $P$ between $9.8 \times 10^{-23}$ and $4.1 \times 10^{-45}$. Patient responses also displayed significantly shorter time courses, with smaller pupillary contraction widths across all protocols (Table 2B). Time to peak values were significantly delayed in glaucoma subjects, the largest and most significant of these effects was elicited by the LSS protocol, which in normal subjects also produced the shortest mean time to peak of the four stimulus protocols (Table 2C).

In addition to these differences associated with glaucoma, significant effects were observed for age across all parameters and protocols, with each 10-year increase in age (DecadeRel60 in Table 2) resulting in significantly smaller amplitudes (between 0.3 and $2.9 \mu \mathrm{m} /$ decade) and longer times to peak. Less-consistent patterns were observed for the effects of a subjects' sex or whether the pupillary response was direct or consensual.

Signal-to-noise ratios for each region varied in a manner similar to response amplitudes, both within and between protocols. Median values (calculated across visual field locations, subjects, eyes, and pupils) for the LSS, LSF, and MSF protocols ranged between $t=2.53$ and $t=2.83$ for normal subjects and between $t=2.07$ and $t=2.30$ for glaucoma subjects. Values were considerably larger for the VSF protocol at $t=4.06$ for normal subjects and $t=3.07$ for those with glaucoma.

ROC curves and areas under the curve (AUCs) were computed for each protocol, for each defect classification, and for different-sized subsets of regions with the greatest deviations from normal ( $n$ worst). ROC analysis offsets the true-positive rate of a diagnostic method (sensitivity) against its false-positive rate $(1-$ specificity), representative ROC curves are shown in Figure 5. AUC values revealed that the VSF stimulus protocol was the most effective at determining a subjects' diagnostic status. Using response amplitudes as a measure, this protocol produced an overall AUC value of $84.8 \% \pm 5.6 \%$ (SE) when all patient eyes were included. Moderate $(n=6)$ and severe $(n=$ 4) eyes produced AUCs of $100 \%$ and eyes with mild defects 

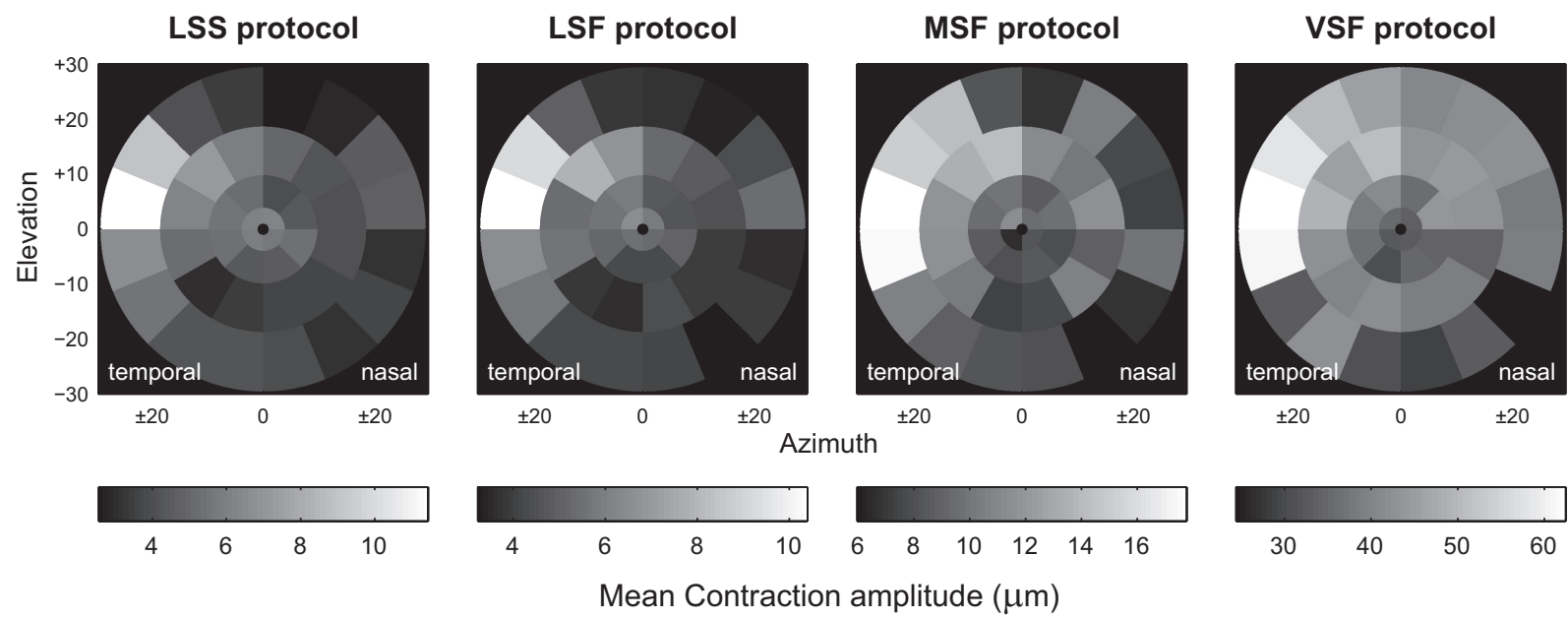

FIGURE 4. Mean regional contraction amplitudes for each stimulus protocol from a multivariate model incorporating age, sex, diagnostic status, and response type (direct or consensual). These regional amplitudes represent the direct responses of male subjects aged 60 with normal vision and are subject to modification by the effects outlined in Table 2 . Left-equivalent visual fields are shown, with temporal regions on the left. In all protocols the most highly responsive regions were located in the temporal hemifield. The range of contraction amplitudes varied between protocols, with the two less sparse protocols (LSP, LSF) producing the smallest contractions and VSF the largest.

$(n=22)$, an AUC of $79.5 \%$ (Table 3, Fig. 5). In general, restricting the analysis to smaller subsets of the most poorly performing regions ( $n$ worst) greatly improved diagnostic accuracy. This effect was particularly apparent in eyes with more pronounced loss and suggests that localized damage was highly diagnostic.

Time to peak and width of responses performed relatively poorly as separate parameters, producing overall AUCs of no more than $70 \%$ in any protocol. A discriminant function incorporating both amplitude and width, however, performed slightly better than amplitude alone in all but the LSS protocol. In the VSF protocol, this produced improved AUCs of $85.5 \% \pm$ $5.0 \%$ (SE) when all patient eyes were included and $81.1 \% \pm$ $7.0 \%$ (SE) for eyes classified as mild. This benefit, however, appeared to be restricted to eyes with less severe defects; amplitude alone produced higher AUCs over a greater range of $n$ worst regions when defects were more severe. A discriminant function combining amplitude with time to peak provided little if any improvement over amplitude alone.

\section{Discussion}

The objective of providing more detailed information regarding the extent of local changes in function was the impetus for the higher-resolution stimulus layout used in this study. The ability to map glaucomatous fields in greater detail is desirable to clinicians; to this end high-resolution versions of both frequency doubling technology perimetry ${ }^{26,27}$ and standard automated perimetry ${ }^{28,29}$ have been developed with some success. For this study, both 40 and 60 region stimulus layouts had been assessed in a preliminary series of experiments on normal subjects ( 9 women, 7 men, aged 27.2 years \pm 9.2 SD). Luminance levels and presentation intervals in this experiment were the same as the VSF protocol, pulses were of 133-ms duration and flickered at $30 \mathrm{~Hz}$. The 60 region protocol had the potential to produce very high-resolution mapping; however, its median SNR, at $t=2.35$ with 16 -second presentation intervals, compared with $t=3.93$ for the 40 region preliminary protocol and $t=4.06$ for the present study VSF protocol, was too low to be practical when using the current methods. Although this value is on par with the less sparse protocols in the study, it presents problems with using faster presentation rates (which tend to reduce SNRs), and in less responsive regions, whether due to inherently low sensitivity or dysfunction. The tendency for low SNR to correlate with high test-retest variability ${ }^{30}$ further reinforces these issues. Given that maximizing SNRs in these less-responsive areas of the visual field is a high priority in our research, only the 40-region layout was assessed with patients. This layout produced SNRs within the range of those of our earlier 24-region/field study, ${ }^{22}$ making it a valid candidate for further investigation.

In the present study, glaucoma subjects' responses were characterized by highly significant reductions in pupillary contraction amplitudes, shorter time-courses and longer times to peak. Perhaps predictably, the overall diagnostic accuracy of individual stimulus protocols in this study was closely linked to the mean amplitudes of responses and apparent differences in these between glaucoma and normal subjects. The best-performing protocols were those in which test pulses were longer and more temporally sparse (i.e., had longer presentation intervals). The VSF protocol, which produced the largest response amplitudes and SNRs in normal subjects and the largest amplitude reductions due to glaucoma also produced the highest ROC AUCs. This protocol's AUC of $85.5 \%$, obtained on inclusion of all patient eyes, is comparable with other perimetric methods: field tests performed on this subject group produced AUCs ranging between $75.9 \%$ and $83.1 \%$ for HFA II and between $85.5 \%$ and $89.1 \%$ for Matrix using Brusini's GSS2 and FDTSS2 $^{31,32}$ and Caprioli's ${ }^{33}$ criteria for abnormality (Fig. 6). Although the less sparse protocols had the advantage of a much greater number of presentations, and thus measurements, for each region: 240 per region for LSS and LSF compared with 15 for VSF, this advantage was not sufficient to increase the precision or confidence with which each mean regional response was estimated and therefore resulted in lower diagnostic accuracy.

A potential confound in pupil perimetry is the tendency of larger responses to become somewhat saturated (i.e., having reached a level where increases in stimulus strength have little effect on response size). Preliminary results from a separate experiment performed by this group have suggested that less saturated responses have a diagnostic advantage (Kolic M, et al. IOVS 2009;50:ARVO E-Abstract 5280; Maddess TL, et al. IOVS 2009; 50:ARVO E-Abstract 5281). It is worth noting that the VSF stimuli that produced the highest AUC values in the present study also presented the highest subjective contrast and elic- 
TABLE 2. Main Effects of Study Variables from a Multivariate Linear Model

A. Amplitude Effects Relative to the Regional Mean Values Shown in Figure 4, $\mu \mathrm{m}$

\begin{tabular}{|c|c|c|c|c|c|c|c|c|c|c|c|c|}
\hline & \multicolumn{3}{|c|}{ LSS Protocol } & \multicolumn{3}{|c|}{ LSF Protocol } & \multicolumn{3}{|c|}{ MSF Protocol } & \multicolumn{3}{|c|}{ VSF Protocol } \\
\hline & $\mathbf{b}$ & $t$ & $\boldsymbol{P}$ & $\mathbf{b}$ & $t$ & $\boldsymbol{P}$ & $\mathbf{b}$ & $t$ & $\boldsymbol{P}$ & $\mathbf{b}$ & $t$ & $\boldsymbol{P}$ \\
\hline Glaucoma & -1.186 & -9.86 & $*$ & -1.360 & -11.33 & $*$ & -2.536 & -10.25 & * & -12.225 & -14.22 & $*$ \\
\hline DecadeRel60 & -0.285 & -4.25 & $*$ & -0.437 & -6.54 & $*$ & -0.587 & -4.26 & $*$ & -2.943 & -6.15 & * \\
\hline Female & -0.431 & -3.56 & 0.0004 & -0.357 & -2.95 & 0.0031 & -0.044 & -0.17 & 0.8616 & 0.188 & 0.22 & 0.8279 \\
\hline Consensual & -0.336 & -2.81 & 0.0050 & -0.425 & -3.56 & 0.0004 & -0.511 & -2.08 & 0.0378 & -1.414 & -1.66 & 0.0979 \\
\hline
\end{tabular}

B. Width Effects Relative to the Mean Widths Shown Below, ms

\begin{tabular}{|c|c|c|c|c|c|c|c|c|c|c|c|c|}
\hline & \multicolumn{3}{|c|}{ LSS Protocol } & \multicolumn{3}{|c|}{ LSF Protocol } & \multicolumn{3}{|c|}{ MSF Protocol } & \multicolumn{3}{|c|}{ VSF Protocol } \\
\hline & $\mathbf{b}$ & $t$ & $\boldsymbol{P}$ & $\mathbf{b}$ & $t$ & $\boldsymbol{P}$ & $\mathbf{b}$ & $t$ & $\boldsymbol{P}$ & $\mathbf{b}$ & $t$ & $\boldsymbol{P}$ \\
\hline Mean width & 252.765 & 427.03 & $*$ & 252.063 & 394.41 & $*$ & 249.818 & 336.19 & $*$ & 276.410 & 612.28 & * \\
\hline Glaucoma & -9.148 & -13.89 & $*$ & -13.250 & -18.64 & $*$ & -11.336 & -13.72 & $*$ & -6.854 & -13.65 & $*$ \\
\hline DecadeRe160 & 5.439 & 14.84 & $*$ & 1.890 & 4.77 & $*$ & 3.358 & 7.30 & $*$ & -2.550 & -9.12 & * \\
\hline Female & 4.416 & 6.65 & $*$ & 5.507 & 7.69 & $*$ & 0.941 & 1.13 & 0.2588 & -2.351 & -4.64 & * \\
\hline
\end{tabular}

C. Time to Peak Effects Relative to the Mean Times to Peak Shown Below, ms

\begin{tabular}{|c|c|c|c|c|c|c|c|c|c|c|c|c|}
\hline & \multicolumn{3}{|c|}{ LSS Protocol } & \multicolumn{3}{|c|}{ LSF Protocol } & \multicolumn{3}{|c|}{ MSF Protocol } & \multicolumn{3}{|c|}{ VSF Protocol } \\
\hline & $\mathbf{b}$ & $t$ & $\boldsymbol{P}$ & b & $t$ & $\boldsymbol{P}$ & $\mathbf{b}$ & $t$ & $\boldsymbol{P}$ & $\mathbf{b}$ & $t$ & $\boldsymbol{P}$ \\
\hline $\begin{array}{l}\text { Mean time } \\
\text { to peak }\end{array}$ & 417.608 & 334.40 & $*$ & 426.906 & 368.38 & $*$ & 432.238 & 282.52 & $*$ & 460.052 & 307.26 & $*$ \\
\hline Glaucoma & 18.817 & 13.55 & $*$ & 7.735 & 6.00 & $*$ & 17.332 & 10.18 & $*$ & 5.552 & 3.33 & 0.0009 \\
\hline DecadeRe160 & 10.066 & 13.02 & $*$ & 9.691 & 13.50 & $*$ & 3.544 & 3.74 & 0.0002 & 2.345 & 2.53 & 0.0115 \\
\hline Female & 8.576 & 6.12 & $*$ & 12.478 & 9.60 & $*$ & 5.354 & 3.12 & 0.0018 & 16.099 & 9.59 & $*$ \\
\hline
\end{tabular}

Comparisons are relative to a constant describing the mean direct responses of male subjects aged 60 with normal vision. For response amplitudes (A), these constants are represented by the regional amplitudes shown in Figure 4. For width (B) and time to peak (C), the effects are relative to the mean values shown in the top row of each set (means across all regions of both eyes and both direct and consensual responses). Units are appropriate for each response characteristic (i.e., amplitude effects are in micrometers, time to peak and width in milliseconds). Effects for consensual responses and differences between regions were estimated only for the amplitude parameter. The $t$ statistics and $P$ values incorporate an adjustment for any correlation between paired eyes. DecadeRe160, mean effect of each 10-year increment over age 60 (for ages less than 60 , the sign of the effect is therefore reversed).

* Significant at $P<5 \times 10^{-5}, d f=5716$, for all comparisons.

ited the largest contraction amplitudes and could potentially have been affected by response saturation. However, we have also observed that saturation becomes less pronounced with increasingly sparse presentation, and it may be that the 16second presentation interval of the VSF protocol has countered this by reducing the summed input to the pupillary response.
The use of flickered stimuli in this experiment did not appear to confer any diagnostic advantage, compare the otherwise similar LSS and LSF protocols (Table 3, Fig. 5). While the reduced contraction amplitudes observed in glaucoma subjects are probably due, at least in part, to the loss or dysfunction of intrinsically photosensitive retinal ganglion cells (ipRGCs), ${ }^{34-38}$
LSS protocol

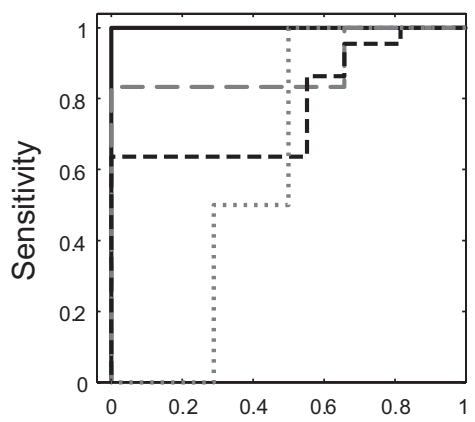

LSF protocol

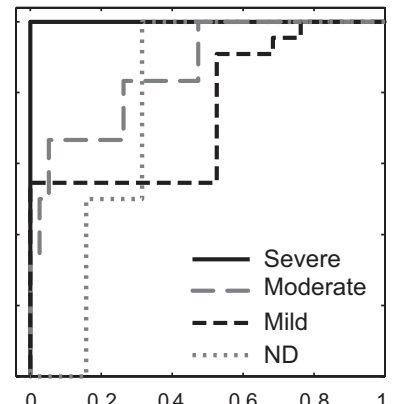

MSF protocol

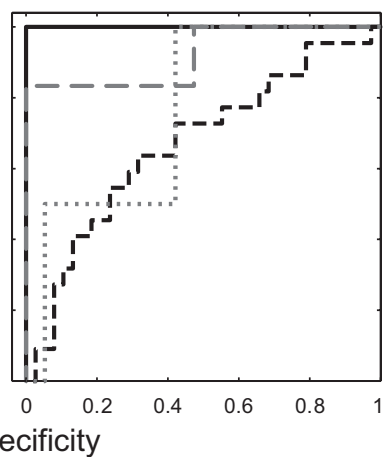

VSF protocol

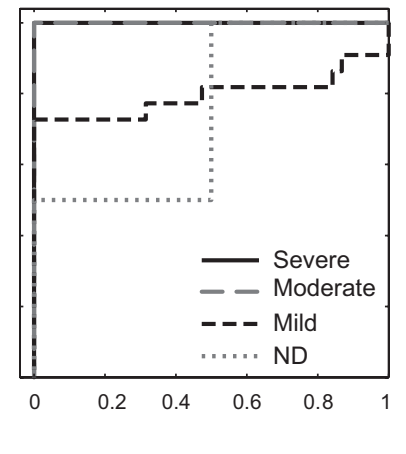

FIGURE 5. ROC plots for each protocol and level of defect obtained using response amplitudes and optimal $n$ worst regions, as outlined in Table 3 . 
TABLe 3. Optimal ROC AUC and AUC SE Values Obtained Using Response Amplitudes and the Parameters Described in the Methods Section

\begin{tabular}{|c|c|c|c|c|c|c|c|c|c|c|c|c|}
\hline \multirow[b]{2}{*}{ Severity } & \multicolumn{3}{|c|}{ LSS Protocol } & \multicolumn{3}{|c|}{ LSF Protocol } & \multicolumn{3}{|c|}{ MSF Protocol } & \multicolumn{3}{|c|}{ VSF Protocol } \\
\hline & AUC & SE & $n$ Worst & AUC & SE & $n$ Worst & AUC & SE & $n$ Worst & AUC & SE & $n$ Worst \\
\hline Severe & 100 & 0 & $1: 3$ & 100 & 0 & $1: 8$ & 100 & 0 & $1: 4$ & 100 & 0 & $1: 7$ \\
\hline Moderate & 89.0 & 10.2 & $1: 2$ & 86.4 & 7.9 & 8:9 & 92.1 & 7.4 & 1 & 100 & 0 & 1 \\
\hline Mild & 77.8 & 6.9 & 1 & 74.3 & 7.1 & 1 & 66.9 & 7.4 & 19 & 79.5 & 7.8 & 1 \\
\hline $\mathrm{ND}^{*}$ & 60.5 & 10.5 & $30: 36$ & 76.3 & 9.7 & $36: 40$ & 76.3 & 14.2 & $39: 40$ & 75.0 & 18.4 & $3: 5$ \\
\hline
\end{tabular}

In general, better results were obtained by restricting the analysis to subsets of those regions with the greatest deviations from normal $(n$ worst). The VSF protocol produced the highest accuracies with AUCs of $100 \%$ for eyes with moderate or severe defects. ROC curves for these results are shown in Figure 5.

* Eye of glaucoma subject with no evidence of disease.

these cells are not the only contributor to the pupillary system. Substantial connections between the striate and extrastriate regions of visual cortex and the pretectal olivary nuclei ${ }^{39}$ form a likely conduit to the pupillary pathway for responses such as those that can be produced in response to flickered stimuli, even in the absence of net luminance increments. ${ }^{40,41}$ Rapidly flickering or transient stimuli dominated by low spatial frequencies, as used in frequency-doubling ${ }^{42,43}$ and flicker perimetry, ${ }^{44,45}$ preferentially stimulate the magnocellular visual pathway, which is responsible for the relaying of motion information. Targeting this pathway allows these techniques to use the low level of redundancy of magnocellular RGCs that results from their large and minimally overlapping receptive fields, ${ }^{46}$ and therefore flickering stimuli may have conferred some benefit. However, the slightly lower performance of the flickered LSF protocol compared with the steady LSS protocol suggest that it is unlikely that any advantage was gained in this study.

In brief, the levels of diagnostic accuracy achieved in this study place it within the range of commonly used screening tests for glaucoma, ${ }^{47}$ and demonstrate the potential of mfPOP

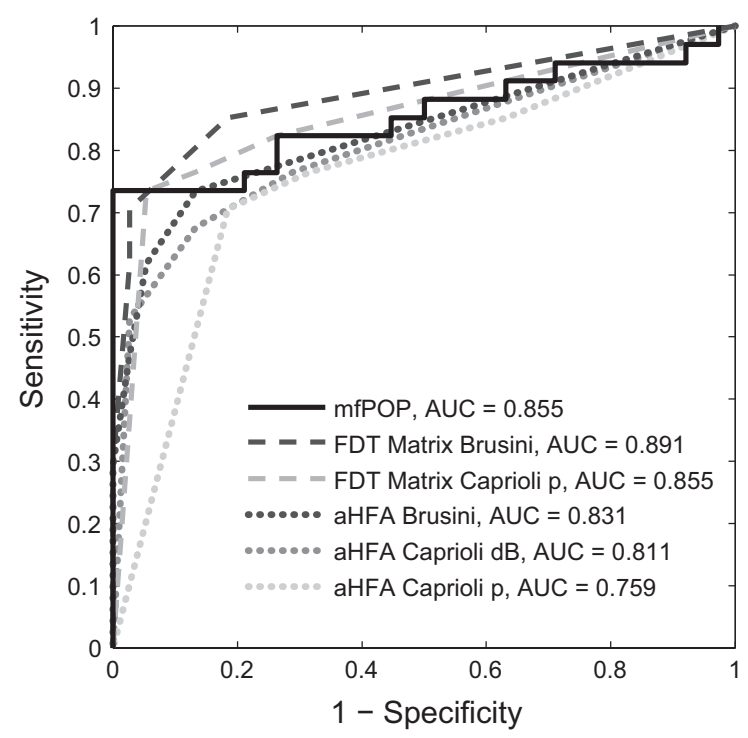

FIGURE 6. Comparison of ROC curves for the mfPOP VSF protocol, FDT matrix 24-2 perimetry and HFA II achromatic SITA-Fast perimetry for the entire subject group. Matrix and aHFA curves were estimated by using Brusini's glaucoma staging systems ${ }^{30,31}$ and Caprioli's strict, moderate, and liberal examples of criteria for abnormality (both $\mathrm{dB}$ and probability map $[P]$ methods). ${ }^{32}$ The mfPOP curve was calculated by using the criteria described in the Methods section. The mfPOP VSF protocol's diagnostic accuracy of AUC $=85.5 \%$ falls slightly above that of aHFA and slightly below that of Matrix for this subject group. as an adjunct to more conventional forms of perimetry. The test is short, having average durations of less than 3 minutes per eye; it is easy to tolerate, and it is less prone to the effects of poorly corrected refraction or lens opacities than many other tests. A high proportion of test data can be used, generally without the need for exclusion of sections containing blinks or losses of fixation. In addition, evidence has been provided that a wide variety of treatments that might be expected to affect pupil function do not markedly affect multifocal pupillary responses at the luminances used here. ${ }^{22}$ Further investigations into intrasubject variability would be beneficial, as would fine-tuning of both stimulus characteristics and analysis methods. These preliminary results, using a 40region high-resolution stimulus, encourage the further development of this technique as a diagnostic tool for glaucoma.

\section{References}

1. Keltner JL, Johnson CA, Anderson DR, et al. The association between glaucomatous visual fields and optic nerve head features in the Ocular Hypertension Treatment Study. Ophthalmology. 2006; 113:1603-1612.

2. Bjerre A, Grigg JR, Parry NR, Henson DB. Test-retest variability of multifocal visual evoked potential and SITA standard perimetry in glaucoma. Invest Ophthalmol Vis Sci. 2004;45:4035-4040.

3. Blumenthal EZ, Sample PA, Berry CC, et al. Evaluating several sources of variability for standard and SWAP visual fields in glaucoma patients, suspects, and normals. Ophthalmology. 2003;110: 1895-1902.

4. Chauhan BC, Johnson CA. Test-retest variability of frequency-doubling perimetry and conventional perimetry in glaucoma patients and normal subjects. Invest Ophthalmol Vis Sci. 1999;40:648656.

5. Heijl A, Lindgren A, Lindgren G. Test-retest variability in glaucomatous visual fields. Am J Ophthalmol. 1989;108:130-135.

6. Spry PG, Johnson CA, McKendrick AM, Turpin A. Variability components of standard automated perimetry and frequency-doubling technology perimetry. Invest Ophthalmol Vis Sci. 2001;42:14041410.

7. Spry PG, Johnson CA, McKendrick AM, Turpin A. Measurement error of visual field tests in glaucoma. Br J Ophthalmol. 2003;87: 107-112.

8. Fankhauser F 2nd, Flammer J. Puptrak 1.0: a new semiautomated system for pupillometry with the Octopus perimeter: a preliminary report. Doc Ophthalmol. 1989;73:235-248.

9. Kardon RH. Pupil perimetry. Curr Opin Ophthalmol. 1992;3:565570.

10. Kardon RH, Kirkali PA, Thompson HS. Automated pupil perimetry: pupil field mapping in patients and normal subjects. Ophthalmology. 1991;98:485- 495.

11. Schmid R, Luedtke H, Wilhelm BJ, Wilhelm H. Pupil campimetry in patients with visual field loss. Eur J Neurol. 2005;12:602-608.

12. Schmid R, Wilhelm B, Wilhelm H. Pupillomotor campimetry in normals. Neuroophthalmology. 2000;23:7-13. 
13. Sutter EE, Tran D. The field topography of ERG components in man, I: the photopic luminance response. Vision Res. 1992;32 433- 446.

14. Wilhelm H, Neitzel J, Wilhelm B, et al. Pupil perimetry using M-sequence stimulation technique. Invest Opbthalmol Vis Sci. 2000;41:1229-1238.

15. Tan L, Kondo M, Sato M, Kondo N, Miyake Y. Multifocal pupillary light response fields in normal subjects and patients with visual field defects. Vision Res. 2001;41:1073-1084

16. James AC. The pattern-pulse multifocal visual evoked potential Invest Opbthalmol Vis Sci. 2003;44:879-890.

17. James AC, Maddess T, Goh XL, Winkles N. Spatially sparse patternpulse stimulation enhances multifocal visual evoked potential analysis. Invest Ophthalmol Vis Sci. 2005;46:3602.

18. Maddess T, James AC, Bowman EA. Contrast response of temporally sparse dichoptic multifocal visual evoked potentials. Vis Neurosci. 2005;22:153-162.

19. Maddess T, James AC, Ruseckaite R, Bowman EA. Hierarchica decomposition of dichoptic multifocal visual evoked potentials. Vis Neurosci. 2006;23:703-712.

20. Ruseckaite R, Maddess T, Danta G, Lueck CJ, James AC. Sparse multifocal stimuli for the detection of multiple sclerosis. Ann Neurol. 2005;57:904-913.

21. James AC, Ruseckaite R, Maddess T. Effect of temporal sparseness and dichoptic presentation on multifocal visual evoked potentials. Vis Neurosci. 2005;22:45-54

22. Maddess T, Bedford S, Goh XL, James AC. Multifocal pupillographic visual field testing in glaucoma. Clin Exp Opbthalmol. 2009;37:678-686.

23. Anderson AJ, Johnson CA. Frequency-doubling technology perimetry and optical defocus. Invest Opbthalmol Vis Sci. 2003;44 4147-4152

24. Bell A, James AC, Kolic M, Essex RW, Maddess T. Dichoptic multifocal pupillography reveals afferent visual field defects in early type 2 diabetes. Invest Opbthalmol Vis Sci. 2010;51:602608 .

25. Efron B. Bootstrap methods: another look at the jackknife. Ann Stat. 1979;7:1-26.

26. Racette L, Medeiros FA, Zangwill LM, Ng D, Weinreb RN, Sample PA. Diagnostic accuracy of the Matrix 24-2 and original N-30 frequency-doubling technology tests compared with standard automated perimetry. Invest Ophthalmol Vis Sci. 2008;49:954-960.

27. Johnson CA, Cioffi GA, Van Buskirk EM. Frequency doubling technology perimetry using a 24-2 stimulus presentation pattern. $O p$ tom Vis Sci. 1999;76:571-581.

28. Westcott MC, McNaught AI, Crabb DP, Fitzke FW, Hitchings RA High spatial resolution automated perimetry in glaucoma. $\mathrm{Br} J$ Ophthalmol. 1997;81:452-459.

29. Westcott MC, Garway-Heath DF, Fitzke FW, Kamal D, Hitchings RA. Use of high spatial resolution perimetry to identify scotomata not apparent with conventional perimetry in the nasal field of glaucomatous subjects. Br J Opbthalmol. 2002;86:761-766.

30. Yang A, Swanson WH. A new pattern electroretinogram paradigm evaluated in terms of user friendliness and agreement with perimetry. Ophthalmology. 2007;114:671-679.

31. Brusini P. Frequency doubling technology staging system 2 . J Glaucoma. 2006;15:315-320.

32. Brusini P, Filacorda S. Enhanced Glaucoma Staging System (GSS 2) for classifying functional damage in glaucoma. J Glaucoma. 2006; $15: 40-46$.

33. Caprioli J. Automated perimetry in glaucoma. Am J Ophthalmol. 1991;111:235-239.

34. Dacey DM, Liao HW, Peterson BB, et al. Melanopsin-expressing ganglion cells in primate retina signal colour and irradiance and project to the LGN. Nature. 2005;433:749-754.

35. Gamlin PD, McDougal DH, Pokorny J, Smith VC, Yau KW, Dacey DM. Human and macaque pupil responses driven by melanopsincontaining retinal ganglion cells. Vision Res. 2007;47:946-954

36. Drouyer E, Dkhissi-Benyahya O, Chiquet C, et al. Glaucoma alters the circadian timing system. PLoS One. 2008;3:e3931.

37. Hannibal J, Hindersson P, Ostergaard J, et al. Melanopsin is expressed in PACAP-containing retinal ganglion cells of the human retinohypothalamic tract. Invest Opbthalmol Vis Sci. 2004;45: 4202-4209.

38. Lagreze WD, Kardon RH. Correlation of relative afferent pupillary defect and estimated retinal ganglion cell loss. Graefes Arch Clin Exp Opbthalmol. 1998;236:401-404.

39. Gamlin PD. The pretectum: connections and oculomotor-related roles. Prog Brain Res. 2006;151:379-405.

40. Drew P, Sayres R, Watanabe K, Shimojo S. Pupillary response to chromatic flicker. Exp Brain Res. 2001;136:256-262.

41. Varju D. Human pupil dynamics. Proceedings of the International School of Pbysics "Enrico Fermi". 1969; Course 43:442- 464.

42. Maddess T, Henry GH. Performance of nonlinear visual units in ocular hypertension and glaucoma. Clin Vision Sci. 1992;7:371383.

43. Maddess T, Goldberg I, Dobinson J, Wine S, Welsh AH, James AC. Testing for glaucoma with the spatial frequency doubling illusion. Vision Res. 1999;39:4258-4273.

44. Weinstein P, Brooser G. Flicker perimetry in glaucoma. Am J Ophthalmol. 1962;53:373-375.

45. Tyler CW. Specific deficits of flicker sensitivity in glaucoma and ocular hypertension. Invest Opbthalmol Vis Sci. 1981;20:204212.

46. Dacey DM, Petersen MR. Dendritic field size and morphology of midget and parasol ganglion cells of the human retina. Proc Natl Acad Sci U S A. 1992;89:9666-9670.

47. Mowatt G, Burr JM, Cook JA, et al. Screening tests for detecting open-angle glaucoma: systematic review and meta-analysis. Invest Ophthalmol Vis Sci. 2008;49:5373-5385. 